\title{
Primary Care Provider Views of the Current Referral-to-Eye-Care Process: Focus Group Results
}

By

Clay D. Holley

\begin{abstract}
A Master's Paper submitted to the faculty of the University of North Carolina at Chapel Hill in partial fulfillment of the requirements for the degree of Master of Public Health in the Public Health Leadership Program.
\end{abstract}

Chapel Hill

2009

Advisor

Second Reader 


\section{MANUSCRIPT:}

A. To be submitted to the Investigative Ophthalmology and Visual Science (IOVS) in July 2009

B. Manuscript to be submitted to an Ophthalmology journal versus Primary Care journal due to the nature of the results, i.e., the results speak more to ophthalmologists to improve their practices than do they speak to primary care providers to improve theirs.

C. Format of following manuscript given as IOVS has requested -1) simple tables that are easy to format into IOVS style (if study is published) and 2) each results table given on a separate page.

D. IOVS does not list a specific upper limit for number of tables or figures submitted. The number of tables will be limited to eight in our submission because a similar focus group manuscript submission by Angela Elam, MD, in 2008 was accepted with a similar format and number of tables. More details for IOVS submission guidelines can be found in the Non-Manuscript Addenda (page 29ff) 


\title{
Primary Care Provider Views of the Current Referral-to-Eye-Care Process: Focus Group Results
}

\author{
Clay D. Holley, BS; Paul P. Lee, MD, JD
}

Author Affiliations: Duke University School of Medicine and the University of North Carolina at Chapel Hill Gillings School of Global Public Health (Mr. Holley); Department of Ophthalmology, Duke University Medical Center (Dr. Lee)

Corresponding Author: Paul P. Lee, MD, JD, Department of Ophthalmology, Duke University Medical Center, DUMC 3802, Durham, NC 27710 (lee00106@mc.duke.edu), Telephone: 919-681-2793, Fax: 919-681-8267

Word count: 3473 
Purpose: To understand the barriers facing primary care providers (PCPs), including nurse practitioners (NPs) and physician assistants (PAs) in the current referral-to-eyecare process and to solicit suggestions from PCPs on how to improve the current referral system.

Methods: We conducted four focus groups with a total of 17 PCPs: two groups with physicians (MDs) - one in a rural setting and one in an academic medical center setting; one group with NPs; and one group PAs. We audiotaped and transcribed all discussions, and both authors performed content analysis of the transcripts with the assistance of qualitative software, NUD*IST Vivo.

Results: The most frequently cited referral barriers include: (1) poor feedback from eye care providers (ECPs), (2) patients' lack of finances/insurance coverage, and (3) difficulty in scheduling an ECP appointment. Among rural PCPs, limited access to ECPs and patients' limited access to transportation also were cited. Suggestions made in all groups on ways to improve the current referral system include: (1) implementing electronic medical records (EMRs), (2) receiving better communication/feedback from ECPs, (3) having ophthalmologists hold clinic days in primary care facilities, and (4) performing retinal scans in primary care clinics.

Conclusions: PCPs desire change(s) in the current referral-to-eye-care system. Better communication between PCPs and ECPs, further implementation of electronic medical records (EMRs), and increasing eye screening in primary care clinics were common themes. Implementing specific suggestions, such as modernizing medical record systems, may help to increase eye care utilization among patients at high risk for advancing eye disease and vision loss. 


\section{INTRODUCTION}

The age 65 years and older $(\geq 65)$ population in the United States is projected to increase from 40 million to 71 million persons in the next 20 years. ${ }^{1}$ Approximately half of those 71 million people will develop glaucoma, age-related macular degeneration, and/or diabetic retinopathy as they age, increasing the need for eye care services. ${ }^{2}$ Regular eye care is associated with better disease outcomes and quality of life for those $\geq 65{ }^{3}$ but almost half of patients with one or more diagnosed eye diseases do not receive eye care according to recommended guidelines. ${ }^{4,5}$ Many patients, particularly those with diabetes, are diagnosed and followed by a primary care provider (PCP) but must be referred to an eye care provider (ECP) to receive eye care. ${ }^{4}$ In the context of this article, we define a PCP as a physician (general internist, family practitioner, general practitioner, or pediatrician), nurse practitioner, or physician assistant who serves as the primary contact point between the patient and the health care system, while an ECP is defined as an ophthalmologist or optometrist.

Based on our review of the literature, little prior work has examined the referral-to-eyecare practices of PCPs. The work that has been done has focused mainly on referral of diabetic patients for diabetic eye examinations, ${ }^{6-12}$ which is an important but singular aspect of the referral-to-eye-care issue. Furthermore, one of these studies ${ }^{7}$ noted that a breakdown in the PCP to ECP referral chain may explain the discrepancy between PCP reported referral-to-eye-care rates and actual rates of ophthalmic examination by an ECP. A study in South Africa also cited referral system issues as a probable cause for many patients not receiving eye care in that country..$^{13}$ As a result, we targeted this PCP to ECP referral step as a point of interest and sought to ask PCPs directly about their 
referral-to-eye-care patterns, the barriers they encounter in that process, and what can be done to improve the referral system.

\section{METHODS}

Prior to the start of this study, approval of the study was granted by the institutional review boards of both Duke University Health System and the University of North Carolina at Chapel Hill (UNC). We first conducted a MEDLINE search surveying literature published in English from 1966 to February 2009, using combinations of key words relevant to PCP referral-to-eye-care patterns and barriers.

After reviewing relevant articles, we realized that very few publications discuss these issues, and most of the ones that do are centered on referral of a patient by an MD, i.e. not an NP or PA, for diabetic eye examinations. We used the information gathered from the literature search to develop a semi-structured script used for the individual interviews and focus groups. To ensure the posed questions were clear and relevant, we pilot tested the script on 3 individuals who did not participate in the focus group study later: one physician (MD), one nurse practitioner (NP), and one physician assistant (PA). We used the revised script to facilitate each focus group discussion to ensure comparability between each group.

We recruited study participants for the academic setting MD, NP, and PA groups from Duke Primary Care (DPC) and UNC Health Care in Durham and Chapel Hill, North Carolina, respectively. The rural MD group was recruited through a personal PCP contact of one of the authors (C.D.H.) in rural southwest Arkansas. We were not able to recruit sufficient numbers of NPs and PAs in rural areas of North Carolina and 
Arkansas to conduct focus groups with those provider types in the rural setting. For the academic setting groups, we used the PCP database of each health care system to collect electronic mail addresses of all PCPs and sent an invitation to each provider individually. For the rural MD group, we sent an electronic mail invitation to one PCP, who then called or personally invited all PCPs practicing in and around a rural town in southwest Arkansas. In all invitations, we asked each PCP to invite other PCPs who met the criteria for participating.

When a PCP expressed interest in joining the study, we confirmed that he/she had primary care experience and either practiced primary care within the academic setting (for academic groups) or the rural setting (for rural MD group). We conducted a total of four focus groups: one academic setting MD group, one rural setting MD group, one academic setting NP group, and one academic setting PA group. We conducted all of the focus groups at fine dining establishments in an area that was easily accessible and familiar to all participants. All participants received a free multi-course dinner $(\$ 75$ approximate value) during the discussion as compensation for their time. Prior to the start of each group, we obtained informed consent from each participant. All of the focus groups were moderated by one of the authors (C.D.H.).

We began each group by expressing appreciation for individuals' participation, stating the objectives and "ground rules" for the focus group, and introductions by the participants. Throughout the discussion, participants were able to speak freely about their ideas on barriers in the referral-to-eye-care system and needed changes to the current referral system. We recorded all of the focus groups with two microcassette tape recorders for the purposes of accuracy and clarity and later transcribed each recording using wordprocessing software. We used qualitative software, NUD*IST Vivo, for content analysis of 
the transcripts. In addition, both authors reviewed and analyzed each of the scripts from individual interviews and focus groups for content and key concepts. The purpose of the current study is to identify a range of issues and ideas on how to improve care delivery that can later be explored quantitatively. Therefore, the results of the focus groups represent all of the information obtained from the discussions, whether it was supported by the majority of the participants or only one.

\section{RESULTS}

\section{Participant Characteristics}

A total of 4 focus groups were conducted. We conducted one group with academic setting MDs, one group with rural setting MDs, one group with academic setting NPs, and one group with academic setting PAs. The size of the focus groups ranged from 4 to 5 people. In all, 17 individuals participated in the groups. Of the 17 participants, a wide range of years of primary care experience was represented (Table 1).

\section{Focus Group Analysis}

A total of 51 comments were made by the focus group participants in regards to barriers to eye care referrals. We classified these comments into 11 different areas of concern (Table 2). The most frequently cited barriers include: (1) poor communication from ECP, (2) patients' lack of finances and/or insurance coverage, (3) difficulty in scheduling ophthalmology appointments, (4) dependence on patient to schedule own appointment, and (5) too many referrals to process in a paper-based system. These five barriers accounted for $80 \%$ of all comments made about barriers in referral-to-eye-care process. Examples of the comments on the most frequent barriers are 
noted in Table 3. In addition to the five most common barriers cited, six other areas were identified as barriers to referral. Table 4 illustrates some of the comments made from each of those areas.

With respect to changes that could be made to the current referral-to-eye-care system, 49 comments in 9 distinct content categories were made by the focus group participants (Table 5). Suggestions on implementing electronic medical records (EMRs) and getting better communication from ECPs were most frequently offered, making up $50 \%$ of the total comments. These comments were categorized similarly to those on barriers. Examples of suggestions from each category are shown in Table 6.

Other data collected show that all PCP types are comfortable treating, (1) uncomplicated eye infections or conjunctivitis and (2) a foreign body amenable to flushing, on their own instead of referring patients with those issues to an ECP. Outside of these two eye/vision problems, most PCPs were not comfortable handling basic visual acuity issues, minor eye trauma, or foreign body of any type. All PCPs except three said that they refer at least one patient to an ECP on a daily basis. Two NPs and one PA said that they refer at least one patient weekly.

Diabetic eye screening was the most commonly cited reason for referral among all PCP types, with cataracts, glaucoma, age related macular degeneration, hypertensive retinopathy, and foreign body also mentioned as common reasons for referral. All PCPs types thought that over $90 \%$ of their referred patients are seen by an ECP when referred for an acute issue, e.g. red, painful eye, but all PCPs except three thought that only 50$70 \%$ of their referred patients are seen for chronic eye or vision issues, e.g. diabetic eye exams. Two NPs thought that $90 \%$ or more of their referred patients are seen for chronic 
issues, while one NP thought that only $40 \%$ were seen. Tables 7 and 8 show responses of PCPs when asked if they would be willing to take on more eye care in their practice than they are performing currently (Table 7) and would they be willing to take on more eye care responsibilities if appropriate reimbursement were available (Table 8). 
Table 1. Participant Characteristics: Number of Participants in Each Year Range of Primary Care Experience.

PCP type

Academic MDs

Rural MDs

NPs

PAs
Years of experience in primary care

$<5$

1

1

1

2

5-10

2

2

2

1

$\begin{array}{cc}11-20 & >20 \\ 1 & 1 \\ 0 & 1 \\ 1 & 0 \\ 1 & 0\end{array}$




\section{Table 2. PCP-cited Barriers in the Referral-to-Eye-Care Process.}

Barriers to eye care referral

No/little feedback from ECP

Patient lack of finances/insurance coverage Difficulty in scheduling ophthalmology appt.

Dependence on patient to schedule own appt. Too many referrals to process in paper-based system

Wait time for patient at ophthalmology office Limited access to ECPs (No full-time ophthalmologist in local area)

Patient access to transportation

Can't understand ECP feedback/too much ophthalmology jargon in notes

PCP time constraints

Patient unwilling to attend ECP appointment
Number of comments by PCP type

Academic Rural

$\begin{array}{ccccc}\text { MDs } & \text { MDs } & \text { NPs } & \text { PAs } & \text { Total } \\ 5 & 1 & 3 & 5 & 14 \\ 3 & 2 & 4 & 4 & 13 \\ 4 & 0 & 2 & 2 & 8 \\ 2 & 0 & 0 & 1 & 3 \\ 3 & 0 & 0 & 0 & 3 \\ 2 & 0 & 0 & 0 & 2 \\ 0 & 2 & 0 & 0 & 2 \\ 0 & 2 & 0 & 0 & 2 \\ 0 & 1 & 1 & 0 & 2 \\ 1 & 0 & 0 & 0 & 1 \\ 0 & 0 & 1 & 0 & 1\end{array}$


Table 3. Examples of Most Frequently Cited Barriers to Referral.

Barrier

Example(s)

No/little feedback from ECP

"Our whole practice has mentioned that we pretty much uniformly don't get communication on the vast majority of cases from the academic eye center, which is pretty huge. I mean, why is that? It's just a block away! And why aren't the ophthalmologists' notes in our EMR? That's another thing I just don't understand." (Academic MD2)

"One of the most challenging things for me is not knowing if the patients ever see the ophthalmologist...You kind of rely on your patients. You're saying, 'When was your last eye exam?', and the patient says, 'Well, I think it was in the spring, but I'm not sure.' You really don't know the outcome of the ophthalmology visit." (PA3)

Patient lack of finances/insurance coverage

Difficulty in scheduling eye appointment

Too many referrals to process in a paper-based system

Dependence on patient to schedule own appointment
"Some of it is financial - if the family can't afford it or their insurance plan doesn't have any kind of vision coverage." (NP1)

"I have to get through the 'phone tree' before there's somebody I can talk to in the ophthalmology office." (NP1)

"There is a big wait if patients just call for themselves for an ophthalmology appointment." (Academic MD2)

"A lot of times the referral person at our front desk just hands the patient a phone number. So, it's on the patient to call and schedule the ophthalmology appointment." (Academic MD5)

"One barrier that's specific to our clinic is the overwhelming number of referrals that we go through in a day, and the staff that has to process them. And it's all paper right now, and things fall through the cracks. And there is no trail. So, it's the workload." (Academic MD3) 
Table 4. Examples of Additional Barriers Identified.

Barrier

Example(s)

Wait time at ophthalmology office

"It's important that the patients don't have to wait two hours in the lobby when they get to the ophthalmologist's office because people kind of get teed off when that happens." (Academic MD1)

Limited access to ECPs

"Well, our local ophthalmologist actually isn't truly local. He comes here once a week or very two weeks, I can't remember...If you have an acute problem, then we find ourselves getting on the phone and calling to figure out if he is in town..." (Rural MD1)

"If it's going to be 4 or 5 days before an ophthalmologist can get my patient in, it affects how I refer patients.” (Rural MD2)

Patient access to transportation

Can't understand ECP feedback

PCP time constraints

Patient unwilling to attend

"With my geriatric patients... a fair number of them can't go or tell me they can't go or 'I don't have a ride' or 'I can't drive down there'." (Rural MD1)

"I just need: 'I saw your patient, diagnosed them with this, and this is what we're doing.' Because if I get a copy of their progress note, and they've got all their [ophthalmology] abbreviations, it's tough to read. The ophthalmology note is the toughest note to read that I can think of." (Rural MD4)

"I don't have time to do [eye screening]. I mean, I could be trained to do it, but I don't have the time to do that." (Academic MD1)

"Well, one barrier is that the patient has to agree to go to the eye care visit." (NP2) 
Table 5. PCP Suggestions for Improving Current Referral-to-Eye-Care System.

Suggestions

Implement electronic medical records

Better communication/feedback from ECPs

Have ophthalmologists in primary care clinic on certain days

Do retinal scans in primary care clinic

Hire ancillary staff in primary care clinic to do

eye screening

Have ophthalmologist in area/town

Have ophthalmology appt. schedule viewable online

More streamlined way to make ophthalmology appts.

Be able to speak to ophthalmologist directly

$\begin{array}{ccccc}\begin{array}{c}\text { Academic } \\ \text { MDs }\end{array} & \begin{array}{c}\text { Rural } \\ \text { MDs }\end{array} & \text { NPs } & \text { PAs } & \text { Total } \\ 5 & 3 & 3 & 2 & 13 \\ 4 & 2 & 2 & 3 & 11 \\ 4 & 1 & 2 & 2 & 9 \\ 2 & 2 & 1 & 2 & 7 \\ 2 & 0 & 0 & 0 & 2 \\ 0 & 2 & 0 & 0 & 2 \\ 2 & 0 & 0 & 0 & 2 \\ 0 & 0 & 0 & 2 & 2 \\ 0 & 0 & 1 & 0 & 1\end{array}$


Table 6. Examples of Comments for Improving Current Referral-to-Eye-Care System.

Suggestion

Implement EMRs

Better communication/feedback from ECPs

Have ophthalmologists in primary care clinic
Example(s)

"I guess in an ideal world if there was just a button I could hit and make it happen. If the EMR is available and it had a button, so I could just push it and the referral would happen. That would be ideal world, I guess. For me, that would be easy." (Rural MD4)

"I would have a patient-centered medical home where we have electronic health records so that everybody in the system can access everything in the system about that patient. That would be 'ideal world'." (NP4)

"I think that number one for me would be getting feedback from the referral." (PA4)

"[From the ophthalmologist] I just need: 'I saw your patient, diagnosed them with this, and this is what we're doing [for treatment].' Because if I get a copy of their progress note, and they've got all their [ophthalmology] abbreviations, it's tough to read. The ophthalmology note is the toughest note to read that I can think of." (Rural MD4)

"Given that a lot of my patients cancel their specialty appointments because they can't afford the \$35 co-pay, I would say: bring the patients in once a month and we have eye clinic at the primary care facility. You, the PCP, do your diabetic visit at the same time [as the eye visit]; it's a $\$ 10$ co-pay or whatever lower co-pay. Bring eye care to the patients...In family practice, I've always viewed us a more of a 'medical home'. I mean, we don't meet all the stipulations through Medicare, but that's how we view ourselves. So, in my mind, that's something [specialty services] that we should provide, and I've worked in practices that have done that. We've brought in specialists to our facility to provide better services for our patients, and it works really well. Our patients are more familiar with us, and so it's much more likely that 
they're going to come and get their care there [at the primary care clinic]." (Academic MD2)

"If you were in practice right down the hall from a really good ophthalmologist that you didn't mind referring patients to, then that would be ideal." (NP4)

"Maybe just have one day that ophthalmologists will come in to the primary care setting, and they could do screenings right there. We could screen a lot of people right there." (PA4)

Do retinal scans in primary care "It would be ideal, because of all our diabetics, to have either the [ophthalmologist] in the practice with us or at least do the retina scan here so that we can be sure that a higher percentage of patients do get things checked.” (Academic MD5)

"They now have these things that you can use to screen for diabetic retinopathy in the primary care office. That might be handy to have; that would be convenient, I think.”(PA3) 
Table 7. PCP interest in performing more eye care than currently performing.

Would you be interested in performing more eye care in your office/clinic than you perform currently?

$\begin{array}{ccccc}\text { PCP type } & & & \\ \text { Academic MDs } & \text { Rural MDs } & \text { NPs } & \text { PAs } & \text { Total } \\ 2 & 2 & 0 & 2 & 6 \\ 0 & 0 & 1 & 2 & 3 \\ 3 & 1 & 0 & 0 & 4 \\ 0 & 0 & 2 & 0 & 2\end{array}$


Table 8. $\mathrm{PCP}$ interest in performing more eye care than currently performing if reimbursement were appropriate.

Would you be interested in performing

more eye care if you were

appropriately reimbursed?

$\begin{array}{ccccc}\text { Academic MDs } & \begin{array}{c}\text { PCP type } \\ \text { Rural MDs }\end{array} & \text { NPs } & \text { PAs } & \text { Total }^{*} \\ 0 & 0 & 1 & 1 & 2 \\ 4 & 0 & 0 & 0 & 4 \\ 2 & 0 & 0 & 4 & 6 \\ 0 & 0 & 1 & 3 & 4 \\ 0 & 3 & 0 & 0 & 3 \\ 0 & 1 & 0 & 0 & 1\end{array}$

No, don't have time

Yes, if staff did it

Yes, if given more time with each

patient

Yes, with appropriate training

Yes

Unsure

0

*Overall number of comments (20) sums to more than number of participants (17) because some participant comments overlapped categories, e.g. PA3 wanting both more time with patients and additional training 


\section{DISCUSSION}

Little prior work has been able to demonstrate the referral-to-eye-care practices of PCPs and what barriers PCPs face in the referral process. The work that has been done has focused mainly on MD (i.e., not NP or PA) referral-to-eye-care patterns for patients with diabetes. ${ }^{6-12}$ Through this pilot study, we sought to identify referral-to-eye-care barriers from the PCP (including NP and PA) perspective and to solicit suggestions from PCPs on how problems in the current referral system can be remedied.

We used the focus group method for several reasons. Focus groups facilitate an atmosphere of self-disclosure in which participants can freely express ideas, opinions, and feelings about a particular topic among a group of people who share similar characteristics. ${ }^{14}$ In our study, all participants shared the experience of providing primary care and navigating the referral system to get their patients seen by ECPs. One of the reasons we separated our focus groups into different provider types (MD, PA, NP) was to maintain a comfortable, permissive environment for all participants, e.g., PAs might be more likely to share freely if they are discussing with other PAs and not with an $\mathrm{MD}(\mathrm{s})$ who could potentially supervise them at work. Another reason for this separation was to determine if any differences in opinion exist between provider types. Focus groups allow a collection of data that present a range of opinions about a particular topic in a rapid and in-depth fashion. ${ }^{15}$ Analysis of the data from three or more focus groups commonly helps identify trends and patterns regarding the issue of interest. ${ }^{14}$

Communication issues underlie many PCP concerns about the current referral-to-eyecare system. In all four focus groups, multiple PCPs cited lack of feedback from ECPs as a barrier in the referral process. Most PCPs said they had trouble getting any kind of feedback about their patients from ECPs, i.e., no phone call, fax, e-mail, note in the 
EMR, or hard copy via mail. Two other PCPs said they receive feedback from ECPs, but the feedback is often difficult to understand because of the ECPs use of "ophthalmology abbreviations and jargon" (rural MD4) or "ophthalmology mumbo jumbo"

(NP1). Our findings are not the first to note poor communication on the part of ECPs ${ }^{13,16}$ and specialists in general. ${ }^{17}$

PCPs in our study suggested that feedback from ECPs be concise and understandable. A suggestion from one PCP was to receive a referral follow-up note from the ECP saying, "I saw your patient, diagnosed them with $\mathrm{X}$, and $\mathrm{Y}$ is what we're doing [for treatment and follow-up]" (rural MD4). This suggestion closely parallels both our own and published ${ }^{18}$ recommendations to ECPs of: 1) returning a report to the PCP in a format understandable to non-ECPs, 2) including in the report results of the eye examination and treatment plan, and 3) adding any recommendations for follow-up. A few PCPs in our study also mentioned that they would like to receive a "no-show" notice from ECPs when their patients do not show up for eye care appointments, which is another published recommendation. ${ }^{18}$

Review articles and studies ${ }^{12,16,18,19}$ identify effective collaboration and communication between PCPs and ECPs as essential to improve delivery of eye care, enhance patients' vision and quality of life, and provide continuity of care. Persistent failure of ECPs to give useful feedback to PCPs could potentially reduce PCP referral-to-eye-care rates or at least alter referral patterns, as one PCP suggested: "Coming here [to an academic medical setting], I was shocked at the lack of communication from the academic ophthalmologists. I've been here for a little over a year, and I have yet to receive any communication...And so, I'm constantly a little tainted. It's not that I tell patients not to go to the academic eye center, but I certainly do not look down upon referring elsewhere 
because I have a higher chance of actually getting communication from an outside provider than one at the academic eye center." (Academic MD2)

The implementation and/or better utilization of EMRs was the most frequently cited suggestion for referral system improvement among the PCPs in our study. Academic PCPs in our study noted that the system in which they work has an EMR, but the ECPs do not properly utilize it, e.g. academic ECPs do not place their progress notes in the EMR for all other providers to access. The use of EMRs could facilitate easier and more consistent communication between PCPs and ECPs and also reduce the difficulty of scheduling eye care appointments, the reliance on patients to schedule their own eye care appointments, and the overwhelming number of referrals to process in a paperbased system, the third, fourth, and fifth most frequently cited barriers in this study, respectively. Academic PCPs suggested that if their EMR system had a function that allows primary care clinics to view the academic eye center's appointment schedule online, scheduling could be completed by the clinic's office staff before patients leave the primary care clinic. This type of scheduling could reduce both paperwork by primary care clinics and reliance on patients to self-schedule appointments after they leave the primary care appointment. Additionally, properly implemented EMRs can provide automated referral reminders to PCPs, which can be important considering the time constraints and complexity of patient issues facing PCPs. ${ }^{10,11,20}$

EMR systems can be expensive and time-consuming to implement and maintain, but almost all PCPs in our study expressed strong support for moving the health care system toward the implementation of EMRs. Even PCPs in the rural area of our study, where practices are not supported by an academic medical system and thus have to pay for and install EMR without outside financial and technical support, were supportive of 
EMRs. In fact, at least two of the four PCPs in the rural MD group had already purchased and implemented EMRs in their own practices despite the cost and time required to do so. With the recent $\$ 19$ billion allocation to the health information technology program of the American Recovery and Reinvestment Act of 2009, EMR implementation could become a more realistic goal in the near future, even for those PCPs practicing in rural and underserved areas. ${ }^{21}$

Patients' lack of finances and/or insurance coverage was the second most frequently identified barrier to referral. This finding is in line with findings from other qualitative studies about overall barriers to eye care (i.e. not solely barriers to referral) with PCPs, ${ }^{12}$ ECPs, ${ }^{12,22}$ and patients. ${ }^{12,22}$ Although PCPs from all focus groups noted this issue as a barrier to referral, few offered suggestions on how to remedy the situation. One PCP, however, suggested the following: "Given that a lot of my patients cancel their specialty appointments because they can't afford the $\$ 35$ co-pay, I would say: bring the patients in once a month and we have eye clinic at the primary care facility. You, the PCP, do your diabetic visit at the same time; it's a $\$ 10$ co-pay or whatever lower co-pay. Bring eye care to the patients. In family practice, l've always viewed us a more of a 'medical home'...So, in my mind, [specialty services] are something that we should provide, and I've worked in practices that have done that. We've brought in specialists to our facility to provide better services for our patients, and it works really well. Our patients are more familiar with us, and so it's much more likely that they're going to come and get their care there [at the primary care clinic].” (Academic MD2)

ECPs holding clinic hours in the same facility as the PCPs was suggested by at least one provider in all four focus groups. PCPs said that it would be much easier to refer to someone who could see patients the same day in the same facility, as was alluded to in 
the previous quote. Alternatively, many but not all PCPs were open to the idea of performing more eye care in their own clinic, with a few stipulations. Most PCPs stated that proper financial and/or temporal reimbursement would have to be in place before they would consider taking on more eye care duties. Academic MDs tended to want technicians or other clinic staff to perform the extra eye care duties. Rural MDs were more willing to take on the duties themselves, while NPs and PAs tended to want more training in eye care before performing extra eye care duties. In fact, NPs, PAs, and some MDs were interested in attending continuing medical education (CME) conferences taught by ophthalmologists to sharpen their eye care skills. For example, PA1 said, "If there would be a hands-on CME [with an ophthalmologist], I would absolutely attend - like a half a day Saturday kind of thing, " to which PA4 said, "I think a lot of mid-levels would attend that."

Participants in the rural MD focus group cited two barriers that were unique to that particular focus group: limited access to ECPs and patients' lack of access to transportation. Rural MDs noted that the "local" ophthalmologist was only in town one to two days per week, which caused them to rely more on referral to a nearby optometrist. Rural PCPs relying more on optometrists than do their non-rural counterparts is a finding that is not unique to our study. ${ }^{8}$ Rural MDs noted that on referrals requiring the immediate attention of an ophthalmologist (e.g., a patient with glaucoma risk factors who presents with a painful, red eye), PCPs must call to several clinics within a 50 mile radius to determine where the "local" ophthalmologist is practicing that day. Rural MDs also mentioned that several of their patients, particularly elderly patients, tell them not to refer them to ECPs because "I don't have a ride to get there," which another study also found in focus groups with patients and ECPs. ${ }^{22}$ Patients often must have family members drive them to medical appointments, and family members do not have the time 
to take their family member to multiple appointments on different days. Thus, the PCP appointment usually takes priority and specialty appointments are not attended (rural MD1).

We note several potential limitations to our study. Focus group analysis carries with it the risk of investigator subjectivity during data analysis. ${ }^{14}$ To avoid this, we used systematic and reproducible methods of coding and categorizing with the use of qualitative software and two-investigator content analysis. Validity and generalizability also are common concerns in focus group studies. ${ }^{14}$ To ensure validity, we pilot tested questions to confirm that they were understood. During focus group discussions, the moderator sought to clarify any areas of ambiguity. In addition, before the end of each focus group, the moderator gave a brief summary of what had been discussed and asked participants to verify the summary comments. As for generalizability, focus groups studies are not intended to generalize due to their nature of going in-depth on a particular topic with a few participants. Instead, the concept of transferability is suggested, which means it is up to the receiver (i.e., you, the reader) to decide if the results can be applied to a different situation. ${ }^{14}$ Generalizability also is limited because few provider characteristics were available for focus group participants in our study.

This pilot study was designed to learn more about the barriers that PCPs face in the referral-to-eye-care process and what suggestions PCPs have to remedy current referral problems. The key findings of this study suggest that PCPs recognize major problems within the current referral system and desire better communication and collaboration with ECPs and better implementation and utilization of EMRs. ECPs can do a better job of providing prompt and understandable feedback to PCPs regarding their referred patients. The recent allocation of funds via the health information technology program of 
the American Recovery and Reinvestment Act of 2009 has the potential to expand EMR systems across the United States, even in rural and underserved areas. PCPs and ECPs should seek out these and other ways to improve continuity of care for the millions of patients at high risk for advancing eye disease and vision loss. The results of this study are currently being explored in a subsequent quantitative study. 


\section{References}

1. U.S. interim projections by age, sex, race, and Hispanic origin. Table 2a: projected population of the United States, by age and sex: 2000 to 2050. 2004.

2. Lee PP, Feldman ZW, Ostermann J, Brown DS, Sloan FA. Longitudinal prevalence of major eye diseases. Arch Ophthalmol 2003;121:1303-1310.

3. Sloan FA, Picone G, Brown DS, Lee PP. Longitudinal analysis of the relationship between regular eye examinations and changes in visual and functional status. $J \mathrm{Am}$ Geriatr Soc 2005;53:1867-1874.

4. Lee PP, Feldman ZW, Ostermann J, Brown DS, Sloan FA. Longitudinal rates of annual eye examinations of persons with diabetes and chronic eye diseases.

Ophthalmology 2003;110:1952-1959.

5. Lee DJ, Lam BL, Arora S, et al. Reported eye care utilization and health insurance status among US adults. Arch Ophthalmol 2009;127:303-310.

6. Wylie-Rosett J, Basch C, Walker EA, et al. Ophthalmic referral rates for patients with diabetes in primary-care clinics located in disadvantaged urban communities. $J$ Diabetes Complications 1995;9:49-54.

7. Dickson PR, McCarty CA, Keeffe JE, Baxter R, Harper CA, Taylor HR. Diabetic retinopathy: examination practices and referral patterns of general practitioners. Med $J$ Aust 1996;164:341-344.

8. Lazaridis EN, Qiu C, Kraft SK, Marrero DG. Same eyes, different doctors: differences in primary care physician referrals for diabetic retinopathy screening. Diabetes Care 1997;20:1073-1077.

9. Lawler FH, Viviani N. Patient and physician perspectives regarding treatment of diabetes: compliance with practice guidelines. J Fam Pract 1997;44:369-373.

10. Kirkman MS, Williams SR, Caffrey HH, Marrero DG. Impact of a program to improve adherence to diabetes guidelines by primary care physicians. Diabetes Care 2002;25:1946-1951.

11. Kraft SK, Marrero DG, Lazaridis EN, Fineberg N, Qiu C, Clark CM, Jr. Primary care physicians' practice patterns and diabetic retinopathy. Current levels of care. Arch Fam Med 1997;6:29-37.

12. Hartnett ME, Key IJ, Loyacano NM, Horswell RL, Desalvo KB. Perceived barriers to diabetic eye care: qualitative study of patients and physicians. Arch Ophthalmol 2005; 123:387-391.

13. de Wet M, Ackermann L. Improving eye care in the primary health care setting. Curationis 2000;23:36-42.

14. Krueger RA, Casey MA. Focus groups: A practical guide for applied research. 3rd ed. Thousand Oaks: Sage; 2000.

15. Daly J, Kellehear A, Gliksman M, Daly KG. The public health researcher: $a$ methodological guide: Oxford University Press; 1997.

16. Li L, Tsang MK, Lau IT, Poon K, Lau E. Assessment of the Quality of Referrals for Diabetic Retinopathy. Assessment 2001.

17. Thind A, Freeman T, Thorpe C, Burt A, Stewart M. Family Physicians' Satisfaction with Current Practice: What Is the Role of Their Interactions with Specialists? Healthc Policy 2009;4:e145-e158.

18. Sinclair SH, Delvecchio C. The internist's role in managing diabetic retinopathy: screening for early detection. Cleve Clin J Med 2004;71:151-159. 
19. Rowe S, MacLean CH, Shekelle PG. Preventing visual loss from chronic eye disease in primary care: scientific review. JAMA 2004;291:1487-1495.

20. Parchman ML, Romero RL, Pugh JA. Encounters by patients with type 2 diabetes - complex and demanding: an observational study. The Annals of Family Medicine 2006;4:40-45.

21. Blumenthal D. Stimulating the adoption of health information technology. $N$ Engl J Med 2009;360:1477-1479.

22. Owsley C, McGwin G, Scilley K, Girkin CA, Phillips JM, Searcey K. Perceived barriers to care and attitudes about vision and eye care: focus groups with older African Americans and eye care providers. Invest Ophthalmol Vis Sci 2006;47:2797-2802. 


\section{NON-MANUSCRIPT ADDENDA:}

\section{RESEARCH QUESTION}

What are the referral practices and barriers to referral for primary care providers (MDs, NPs, \& PAs) to ophthalmologic examination for patients age 65 years or older?

\section{$\underline{\text { HYPOTHESIS }}$}

We expect primary care providers to refer patients to eye care only as their busy schedules allow. We hypothesize that time constraints during patient visits will be a commonly noted barrier to referral by PCPs. We expect most PCPs to be using a paperbased system for referrals, and PCPs will be aware of the current inadequacies in the referral system.

\section{$\underline{\text { Addendum to Introduction (pages 5-6) }}$}

The seven studies listed (see references 6-12 in manuscript) were the only studies even superficially discussing PCP referral-to-eye-care practices in our extensive review of the literature and subsequent review of reference lists from relevant papers. The fact that little prior work has been done on this topic was one of the main reasons my Duke mentor, Paul Lee, and I chose to pursue this project. Hopefully we are beginning a foundation of knowledge on this topic that can be built upon by us and other researchers to discover the main problems and issues in the current PCP referral-to-eye-care system and how to remedy those problems. 


\section{Addendum to Methods (see page 6ff)}

Systematic Review of the Literature:

We first reviewed the literature on primary care provider referral practices to eye care between 1966 and October 2008. The initial MEDLINE search terms were the following: “primary care provider AND eye referral"; "primary care provider AND diabetes AND eye exam"; "primary care AND eye"; "primary care AND referral AND eye"; "eye AND referral pattern"; "PCP AND eye AND referral"; "PCP AND referral"; "PCP AND referral AND factors AND eye". After those searches yielded very few potentially relevant articles, we used the following MEDLINE search terms a few months later to search for literature between 1966 and February 2009: "referral process AND primary care AND eye"; "referral practices AND eye"; "primary care AND referral AND eye"; "PCP diabetes eye referral"; and a repeat of several search terms from the original search terms listed above. Additionally, we performed two Google Scholar searches using the terms "primary care AND referral AND eye" and "PCP diabetes eye referral" and used the Google Scholar function of identifying more recent articles that have cited relevant articles. We also examined relevant article reference lists for other potentially relevant studies.

We reviewed abstracts for citations in peer-reviewed journals, and because of the paucity of relevant studies, did not place any limits on the MEDLINE search terms. The initial searches were low yield and resulted in only two directly applicable articles (see Lazardis $^{8}$ and Lawler ${ }^{9}$ studies in manuscript reference list). For example, the first search with terms "primary care provider AND eye referral" yielded 6 articles, only one of 
which (Lawler) was included, leaving five articles unused. As a result, we expanded our MEDLINE search to an analogous topic, i.e., primary care referral of diabetic Medicare patients to foot care under the search terms "diabetes AND referral AND medicare", "referral AND diabetes AND foot care", etc., but these searches failed to yield relevant studies. Finally, we searched MEDLINE for referral practices of PCPs in general using the search terms "referral AND PCP AND patterns", "referral AND PCP AND practices", and "primary care AND referral" and the aforementioned Google Scholar searches. These searches along with a reference list search yielded five additional applicable articles (see Wylie-Rosett ${ }^{6}$, Dickson $^{7}, \operatorname{Kirkman}^{10}, \mathrm{Kraft}^{11}$, and Hartnett ${ }^{12}$ articles in manuscript reference list). For a detailed list of systematic search terms and limits, please refer to Table 11 in the Appendix.

We excluded the following: studies that used the words "primary care" and "referral" and "eye care" or similar terms but did not actually assess 1) factors or barriers affecting the referral process or 2) potential solutions for the deficiencies of the current system; studies that assessed the referral pattern between primary care eye-specific providers, i.e., optometrists, and ophthalmologists. Because of the small number of studies assessing referral practices of PCPs, we included all peer-reviewed studies, including case reports and case series, that listed even one factor, barrier, or remedy involving the primary care to eye care referral axis. The checklist for inclusion and exclusion criteria is found in Table 12 of the Appendix. Evidence grades were not assessed for the selected articles. 


\section{Addendum to Results (see pages 8-19)}

Table 9. How often PCPs see \& refer patients with eye/vision problems

$\begin{array}{lcccc}\text { PCP type } & \text { frequency of referral to ECP } & \text { No } \\ & & \text { Daily } & \text { Weekly } & \text { answer } \\ \text { Multiple/day } & 0 ; 5 & 0 ; 0 & 1 ; 0 \\ \text { Academic MDs } & 4 ; 0 & 0 ; 3 & 0 ; 0 & 0 ; 0 \\ \text { Rural MDs } & 4 ; 0 & 1 ; 3 & 0 ; 2 & 3 ; 0 \\ \text { NPs } & 0 ; 0 & 2 ; 2 & 0 ; 1 & 0 ; 0 \\ \text { PAs } & 2 ; 1 & & \end{array}$

Table 10. Eye/vision problems that PCPs would treat without ECP intervention

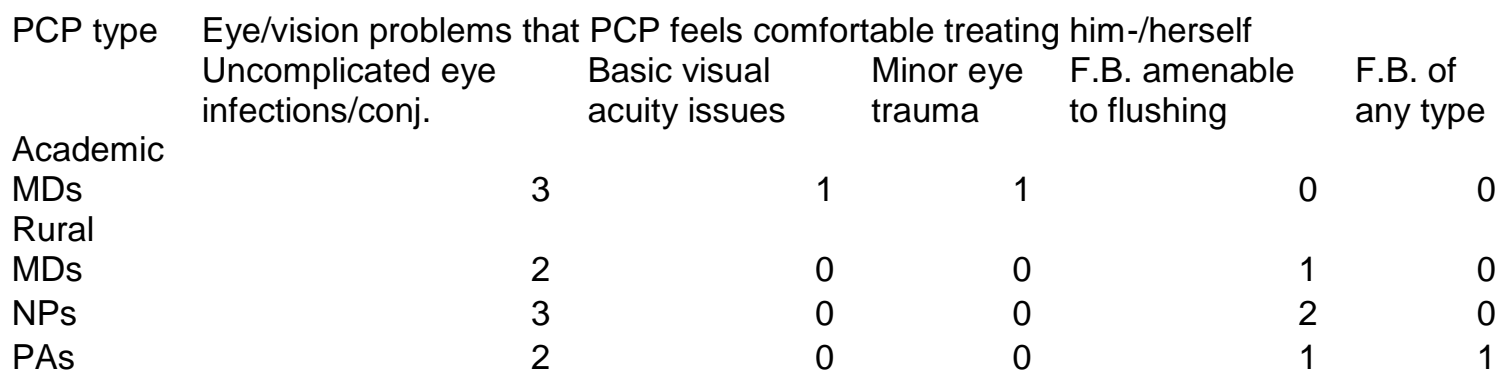

\section{Addendum to Discussion (see pages 20-26)}

\section{Focus Groups as a Research Method}

Qualitative research methods are well-suited for exploring subjective views on an issue.

They put the subject and its perception of the world at the center of their attention, and the lived experiences of those studied or their accounts of it serve as the basis for data analysis. Additionally, a qualitative approach allows the illustration of particular types of primary care provider referral practices to eye care and documents different foci referral practices between the four groups (academic MDs, rural MDs, PAs, NPs) questioned. With this in mind, referral practices were explored in focus groups with primary care MDs, NPs, and PAs from academic settings and with MDs from a rural setting. Focus 
groups were not held with NP and PA groups in rural settings because insufficient numbers of those PCP types were recruited in the recruitment phase of this study.

Focus groups are commonly used as a form of qualitative research method in the sociology of health and illness. Compared with individual interviews, focus groups allow access to research participants who may find one-on-one, face-to-face interaction intimidating or uncomfortable ${ }^{1}$. Additionally, focus groups can create multiple lines of communication and offer participants a safe environment where they can share experiences, practices, ideas, and beliefs in the company of people which have common experiences in clinical practice. The resulting atmosphere of familiarity is helpful in facilitating statements from focus group participants. The interaction among group participants often reduces the amount of interaction between the moderator and the individual members of the group, and the dynamics within the group decrease the influence of the researcher over the interview process and thereby give a more prominent role to the participants' opinions ${ }^{2}$.

The group setting has a synergistic effect in stimulating thinking and verbal contributions. Less direct methods such as focus groups are considered as more appropriate to elicit responses that better reflect the social realities of the interviewees ${ }^{3}$. With particular reference to our research objective to explore particular referral practices of primary care providers to eye care and in order to incorporate the experiences of those practicing primary care into survey questions, focus groups were chosen as the appropriate method for obtaining the information we sought. 
Focus groups are group discussions with 8-12 participants in most cases, but one of us (Paul P. Lee) who has extensive experience in conducting focus groups has found that 46 participants is optimal for productive, meaningful discussion. The moderator addresses a number of issues (foci) for discussion and ensures that the discussion remains on the subject of interest. Otherwise, interference with the discussion is kept to a minimum, which is motivated by the aim to create a communication situation which closely resembles a natural interaction ${ }^{3}$. In the present study, group participants were asked to discuss what types of barriers prevent them from referring their primary care patients to an eye care provider and what ideas they had for potential solutions to those barriers. We developed a set of focus group guidelines for the moderator that included probes designed to re-focus the discussion if necessary.

\section{Advantages/Disadvantages of Qualitative Research}

Qualitative research methods are often described as more naturalistic, anthropological, observational, and interpretive than are quantitative research methods. Qualitative methods are broad in scope and thus are difficult to define precisely but have been described as inquiries outside the framework prescribed by the scientific method ${ }^{4}$. Qualitative research methods include but are not limited to: case studies, grounded theory, life histories, hermeneutics, and participant observer research. In qualitative research, researchers attempt to capture data from subjects within the context of their natural settings, e.g., a group of people sitting around a table discussing politics. Detailed data are captured through open-ended (and a few close-ended) questions using techniques like historical analysis, focus groups, interviews, surveys, questionnaires, and diaries ${ }^{5}$. 
Qualitative research generally does a better job than quantitative research at reaching a greater level of depth and detail into the topic of interest. Because of the greater depth, however, fewer subjects tend to be studied, and the result is a reduction in generalizability. Qualitative methods tend to create an environment in which all subjects can feel free to openly discuss new ideas and generate new theories. Participants can discuss issues that are important to them instead of answering closed questions, and they can also clarify ambiguities in the questions or discussion topics. Some participants, however, may feel that the openness of the interaction is uncomfortable because of personality, gender, race/ethnicity or various other differences between him/her and the researcher, and the result can be altered answers and discussion during the qualitative sessions ${ }^{3,5}$.

Qualitative methods can yield results that are difficult to replicate due to lack of structured design or standardized procedures. Also, because the researcher often interacts personally with the subjects, the data can be biased and actually reflect the view of the researcher(s) instead of the participating subjects. Personal bias can be avoided if the researcher uses a third party to gather data, e.g., hiring a moderator to conduct focus groups ${ }^{3}$. Primary because of monetary constraints, we did not hire a moderator to conduct our focus groups. 


\section{Lessons Learned from Conducting Focus Groups (that I did not find in published sources)}

Conducting focus groups with PCPs was challenging. Most PCPs are time constrained and understandably hesitant to give a piece of their limited personal time to participate in a research study. While planning the first focus group with academic MDs, I learned to over-recruit for each group. For example, I originally had seven primary care MD's signed up for the first focus group, but one of these cancelled at the last minute and one did not show up for the focus group. For subsequent groups, I over-recruited by two to four PCPs, more than the suggested over-recruitment of one to two ${ }^{3}$, to ensure that I had at least four participants in each group. As I anticipated, one or more potential participants cancelled or failed to show up for each of the four groups, making me glad that I had over-recruited.

I also learned to always have a plan B (and plan C). With each group, I tried to have a plan for an alternate location, time, and/or date in the case that some unforeseen circumstance presented itself. For example, I originally planned the rural MD focus group for a location in the mountains of North Carolina. The restaurant location, menu, and tentative date and time had been set up weeks in advance, but my recruiter, an MD who works in the area of the planned focus group, kept forgetting to recruit other potential participants. Despite several reminder phone calls, he never was able to recruit more than one other MD. As a result, I had to move to plan $\mathrm{B}$, which was to conduct the focus group with a group of rural physicians in Arkansas. I was able to conduct this 
focus group in December, just before I left the country for a study abroad trip. If I had not had this plan B, rural MDs would not have been represented in this study.

\section{ACKNOWLEDGEMENTS}

My sincere thanks to the following people for their assistance with this study and paper:

- My wife, Esma, who has never failed to support me through good times and bad and provided much needed assistance with organizing and conducting the focus groups

- My Mom and Dad, Roger and Kay, whose prayers and support are always present on my behalf, even when their problems have been bigger than my own

- Paul Lee, MD JD, who has been understanding with my unexpected delays and has provided and continues to provide invaluable mentorship

- Margaret Gourlay, MD MPH, who patiently provided suggestions and editing

- Diane Calleson, $\mathrm{PhD}$, who provided support, understanding, and advice during the tough times of the past 18 months 


\section{APPENDIX:}

Table 11 (Appendix Item 1). Systematic Review Literature Searches

\begin{tabular}{|c|c|c|c|c|c|}
\hline Date & Database & Main search terms & Modifiers & Yield & $\begin{array}{c}\text { Used } \\
\text { search? }\end{array}$ \\
\hline $2 / 20 / 08$ & Medline & $\begin{array}{c}\text { Primary care } \\
\text { provider AND eye } \\
\text { referral }\end{array}$ & English & 6 articles & $\begin{array}{l}\text { Yes; Lawler } \\
\text { article }\end{array}$ \\
\hline $2 / 20 / 08$ & Medline & $\begin{array}{c}\text { primary care } \\
\text { provider AND } \\
\text { diabetes AND eye } \\
\text { exam }\end{array}$ & English & 5 & no \\
\hline $12 / 8 / 08$ & Medline & $\begin{array}{l}\text { Primary care AND } \\
\text { referral AND eye }\end{array}$ & none & 259 & Yes \\
\hline $12 / 10 / 08$ & Medline & $\begin{array}{l}\text { Eye AND referral } \\
\text { pattern }\end{array}$ & none & 76 & no \\
\hline $12 / 11 / 08$ & Medline & $\begin{array}{l}\text { PCP AND eye AND } \\
\text { referral }\end{array}$ & none & 73 & $\begin{array}{l}\text { Yes - } 2 \\
\text { articles }\end{array}$ \\
\hline $12 / 13 / 08$ & Medline & PCP AND referral & none & $\begin{array}{c}3238 \\
{\text { (reviewed } 1^{\text {st }}} \\
60 \text { articles) } \\
\end{array}$ & No \\
\hline $12 / 17 / 08$ & Medline & $\begin{array}{c}\text { PCP AND referral } \\
\text { AND factors AND } \\
\text { eye }\end{array}$ & none & 15 & $\begin{array}{l}\text { Yes - one } \\
\text { article }\end{array}$ \\
\hline $12 / 27 / 08$ & Medline & $\begin{array}{c}\text { Referral process } \\
\text { AND primary care } \\
\text { AND eye }\end{array}$ & none & 14 & $\begin{array}{c}\text { Yes- } \\
\text { duplicate } \\
\text { article }\end{array}$ \\
\hline $12 / 27 / 08$ & Medline & $\begin{array}{c}\text { Referral practices } \\
\text { AND eye }\end{array}$ & none & 81 & $\begin{array}{c}\text { Yes- } \\
\text { duplicate } \\
\text { article }\end{array}$ \\
\hline $12 / 27 / 08$ & $\begin{array}{l}\text { Google } \\
\text { Scholar }\end{array}$ & $\begin{array}{l}\text { Primary care AND } \\
\text { referral AND eye }\end{array}$ & none & $\begin{array}{c}57,600 \\
{\text { (reviewed } 1^{\text {st }}} \\
40 \text { articles) }\end{array}$ & $\begin{array}{l}\text { No (all } \\
\text { repeats from } \\
\text { Medline } \\
\text { searches) }\end{array}$ \\
\hline $04 / 07 / 09$ & $\begin{array}{l}\text { Google } \\
\text { Scholar }\end{array}$ & $\begin{array}{l}\text { PCP diabetes eye } \\
\text { referral }\end{array}$ & none & & $\begin{array}{c}\text { Yes - } \\
\text { duplicate } \\
\text { article } \\
\end{array}$ \\
\hline
\end{tabular}


Table 12 (Appendix Item 2). Inclusion/Exclusion Checklist (adapted from Cook et al. ${ }^{6}$ )

\begin{tabular}{|c|c|c|c|c|}
\hline \multicolumn{5}{|l|}{ Citation } \\
\hline $\begin{array}{l}\text { Level of } \\
\text { Review: }\end{array}$ & Title __ & Abstract & Article _ & Date: ____L \\
\hline \multicolumn{5}{|c|}{ Selection Criteria Met } \\
\hline \multicolumn{3}{|c|}{$\begin{array}{l}\text { Population: Does the study specifically } \\
\text { address primary care providers'practice? }\end{array}$} & _ yes & no \\
\hline \multicolumn{3}{|c|}{$\begin{array}{l}\text { Setting: Does the study specifically } \\
\text { discuss referral to eye care from primary } \\
\text { care? }\end{array}$} & $\ldots$ yes & no \\
\hline \multicolumn{3}{|c|}{$\begin{array}{l}\text { Measures: Does the study specifically } \\
\text { discuss barriers in the referral-to-eye } \\
\text { care system and/or ways it can be } \\
\text { improved? }\end{array}$} & _ yes & no \\
\hline \multicolumn{5}{|c|}{ Action: __ include _ _ exclude } \\
\hline \multicolumn{5}{|c|}{ Reasons for exclusion: } \\
\hline
\end{tabular}

Appendix Item 3. Investigative Ophthalmology and Visual Science manuscript guidelines

\section{A. Manuscript Preparation}

Structure: the main manuscript document should be organized as follows:

a. Title Page

b. Structured Abstract: 250-word limit

c. Text

d. Acknowledgments

e. References

f. Figure legends, tables, and figures, if not embedded in text

Pages should be numbered.

1. Title Page

The title page, which must be part of the main manuscript file, should include the title, authors' names and institutions, and other manuscript information such as word count and grant information. 
The title must contain no more than 150 characters, including punctuation and spaces.

2. Structured abstract

A structured abstract of fewer than 250 words is required for articles and should be arranged under the following headings: Purpose, Methods, Results, Conclusions. Define abbreviations at first mention, and do not include references. The abstract must be included as part of the main manuscript file.

3. Text

IOVS recommends a 3,500 or fewer word count, excluding title page, legends, and references. The text should be double-spaced.

In a brief Introduction (don't use any subheadings), provide the research rationale and objectives without extensively reviewing the literature.

In the Methods section, describe the experimental design, subjects used, and procedures followed. Previously published procedures should be identified by reference only. Provide sufficient detail to enable others to duplicate the research. Use standard chemical or nonproprietary pharmaceutical nomenclature. In parentheses, identify specific sources by brand name, company, city, and state or country.

If human subjects were involved in the investigation, the Methods section must confirm that: (1) the research followed the tenets of the Declaration of Helsinki; (2) informed consent was obtained from the subjects after explanation of the nature and possible consequences of the study; and (3) where applicable, the research was approved by the institutional human experimentation committee or institutional review board (IRB).

If experimental animals were used in the investigation, the Methods section must confirm adherence to the ARVO Statement for the Use of Animals in Ophthalmic and Vision Research and, where applicable, approval by the appropriate IRB.

Present the Results with a minimum of discussion. Cite all tables and figures in numerical order.

Limit the Discussion to statistically significant data and their limitations. Do not reiterate results. 
Please review your manuscript carefully prior to submission. Authors needing or seeking assistance with English grammar and usage may utilize the IOVS Volunteer Editor Program (see http://www.iovs.org/misc/voleds.shtml).

4. Acknowledgments

Acknowledgments should be written in the third person and be limited to colleagues and research assistants. Acknowledgments are not meant to recognize appreciation for personal or manuscript production support. Including dedications to individuals or groups is not permitted by IOVS journal policy.

\section{References}

List references numerically by order of citation in the text, not alphabetically. All references must be cited in the text or tables, shown as superscript numbers. Authors are responsible for the accuracy of references.

- Unpublished data (including material in preparation or submitted) or personal communications should be listed parenthetically in the text only with year received or recorded.

- References to journal articles should include (1) author(s) (if there are more than six, write "et al." after the third name), (2) title, (3) journal name (as abbreviated in Index Medicus), (4) year, (5) volume number, and (6) inclusive page numbers.

- References to books should include (1) author(s), (2) chapter title (if any), (3) editors (if any), (4) title of book, (5) city of publication, (6) publisher, (7) year, and (8) inclusive page numbers.

- ARVO abstract citations are to appear parenthetically within the text, not as bibliographic references. For ARVO abstracts from 1977 to 2001, citations should include (1) name of first author, (2) "IOVS", (3) year, (4) volume number, (5) "ARVO Abstract", and (6) program number. For ARVO abstracts from 2002 forward, citations should include (1) name of first author, (2) "IOVS", (3) year, (4) volume number, (5) "ARVO E-Abstract", and (6) program number.

- Reviewers are not required to look up online website references.

Examples: 


\title{
Journals
}

Choudhury A, Palkanis VA, Bowers WE. Characterisation and functional activity of dendritic cells from rat choroid. Exp Eye Res. 1994;59:297-304.

\section{Books}

Stryer L. Biochemistry. 2nd ed. San Francisco, CA: WH Freeman; 1981:559-596.

\author{
Abstracts \\ 1977-2001: (Otaishat NM, et al. IOVS 1997;38:ARVO \\ Abstract 1417) \\ 2002- : (Roska BM, et al. IOVS 2002;43:ARVO E-Abstract \\ 1415)
}

6. Tables, legends, figures, movies

a. Tables must be included in the main manuscript file. Each table should have a brief, self-contained title, understandable without reference to the text. Assign a short heading to each table column. Footnotes in tables should use symbols in the following

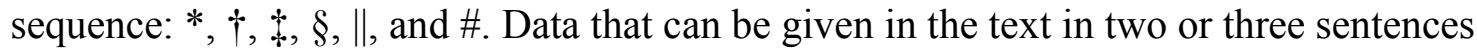
should not be presented in table format.

b. Legends should sum up the intent and content of the data contained in the figure. Use complete sentences or noun phrases with necessary modifiers, and conclude with a period.

c. Figures should be cited in the text, in numerical order using Arabic numerals. Figures may be placed within the main manuscript file or uploaded separately. If a figure contains multiple parts, it should be assembled on one page; Figures 1A and 1B should not appear on separate pages. Please label each figure appropriately just beneath the inserted image. For example, labels should read "Fig. 1" or "Figure 1."

In the event that your manuscript is accepted, the Editorial Office will require you to upload your figures as TIFF or EPS files for the printer. Therefore, while any type of file may be embedded within the manuscript file, it is recommended that graphics be prepared using a program which can save files in a format that can ultimately be saved and submitted as EPS or TIFF. In order to achieve the best quality graphic in the printed version of IOVS, graphics should be saved in CMYK (Cyan, Magenta, Yellow, Black) rather than RGB (Red, Green, Blue). The resolution specification for TIFF and EPS files is $1200 \mathrm{dpi}$ for monochrome, such as lineshots that are black and white only; 300 dpi for gray/CMYK, such as black and white or 
color photographs; and 600 dpi for combinations, such as photographs labeled with letters or other markings containing thin lines. For more detail regarding digital graphics, see http://cpc.cadmus.com/da/guidelines.asp. Authors of accepted manuscripts can see http://www.iovs.org/misc/accepted.shtml for further details regarding figure requirements for publication.

d. Movies can be saved as QuickTime files. Since movies can only be viewed online, a movie may be linked to an image (for example, a frame or frames from the movie) that appears as a figure in the manuscript. 


\section{References for Addenda/Appendix}

${ }^{1}$ Morgan DL. Focus groups as qualitative research. Newbury Park, CA: Sage; 1988.

${ }^{2}$ Madriz E. Focus groups in feminist research. In Handbook for Qualitative Research, 2nd ed. (Denzin NK \& Lincoln YS, eds.), London: Sage, pp. 835-850; 2000.

${ }^{3}$ Krueger RA, Casey MA. Focus groups: a practical guide for applied research. 3rd ed. Thousand Oaks: Sage; 2000.

${ }^{4}$ Byrne MM. Evaluating the findings of qualitative research. AORN J. 2001 Mar;73(3):703-6.

${ }^{5}$ Perecman E \& Curran SR, eds. A handbook for social science field research: essays and bibliographic sources on research design and methods. Thousand Oaks: Sage; 2006.

${ }^{6}$ Cook DJ, Mulrow CD, Haynes RB. Systematic reviews: synthesis of best evidence for clinical decisions. Ann Intern Med 1997 Mar 1;126(5):376-380. 